\title{
Constrained Synchronization and Subset Synchronization Problems for Weakly Acyclic Automata
}

\author{
Stefan Hoffmann ${ }^{[0000-0002-7866-075 X]}$ \\ Informatikwissenschaften, FB IV, Universität Trier, Universitätsring 15, 54296 Trier, \\ Germany, hoffmanns@informatik. uni-trier.de
}

\begin{abstract}
We investigate the constrained synchronization problem for weakly acyclic, or partially ordered, input automata. We show that, for input automata of this type, the problem is always in NP. Furthermore, we give a full classification of the realizable complexities for constraint automata with at most two states and over a ternary alphabet. We find that most constrained problems that are PSPACE-complete in general become NP-complete. However, there also exist constrained problems that are PSPACE-complete in the general setting but become polynomial time solvable when considered for weakly acyclic input automata. We also investigate two problems related to subset synchronization, namely if there exists a word mapping all states into a given target subset of states, and if there exists a word mapping one subset into another. Both problems are PSPACE-complete in general, but in our setting the former is polynomial time solvable and the latter is NP-complete.
\end{abstract}

Keywords: automata theory $\cdot$ constrained synchronization $\cdot$ computational complexity $\cdot$ weakly acyclic automata $\cdot$ subset synchronization

\section{Introduction}

A deterministic semi-automaton is synchronizing if it admits a reset word, i.e., a word which leads to a definite state, regardless of the starting state. This notion has a wide range of applications, from software testing, circuit synthesis, communication engineering and the like, see 2830. The famous Černý conjecture [7] states that a minimal length synchronizing word, for an $n$-state automaton, has length at most $(n-1)^{2}$. We refer to the mentioned survey articles 28130] for details. 1 .

Due to its importance, the notion of synchronization has undergone a range of generalizations and variations for other automata models. In some generalizations, related to partial automata [22, only certain paths, or input words, are allowed (namely those for which the input automaton is defined).

\footnotetext{
${ }^{1}$ A new and updated survey article (in Russian) is currently in preparation by Mikhail V. Volkov 29].
} 
In 15] the notion of constrained synchronization was introduced in connection with a reduction procedure for synchronizing automata. The paper 13 introduced the computational problem of constrained synchronization. In this problem, we search for a synchronizing word coming from a specific subset of allowed input sequences. For further motivation and applications we refer to the aforementioned paper 13. In this paper, a complete analysis of the complexity landscape when the constraint language is given by small partial automata with up to two states and an at most ternary alphabet was done. It is natural to extend this result to other language classes, or even to give a complete classification of all the complexity classes that could arise. For commutative regular constraint languages, a full classification of the realizable complexities was given in [16. In [17, it was shown that for polycyclic constraint languages, the problem is always in NP.

Let us mention that restricting the solution space by a regular language has also been applied in other areas, for example to topological sorting [1, solving word equations 910 , constraint programming 23, or shortest path problems 24]. The road coloring problem asks for a labeling of a given graph such that a synchronizing automaton results. A closely related problem to our problem of constrained synchronization is to restrict the possible labeling(s), and this problem was investigated in 32 .

In 13 it was shown that we can realize PSPACE-complete, NP-complete or polynomial time solvable constrained problems by appropriately choosing a constraint language. Investigating the reductions from [13, we see that most reductions yield automata with a sink state, which then must be the unique synchronizing state. Hence, we can conclude that we can realize these complexities with this type of input automaton.

Contrary, for example, unary automata are synchronizing only if they admit no non-trivial cycle, i.e., only a single self-loop. In this case, we can easily decide synchronizability for any constraint language in polynomial time. Hence, for these simple types of automata, the complexity drops considerably. So, a natural question is, if we restrict the class of input automata, what complexities are realizable?

Here, we will investigate this question for the class of weakly acyclic input automata. These are automata such that the transition relation induces a partial order on the state sets. We will show that for this class, the constrained synchronization problem is always in NP. Then, in the spirit of the work [13], we will give a full classification of the complexity landscape for constraint automata with up to three states and a ternary alphabet. Compared with the classification result from [13, we find that most problems that are PSPACE-complete in general will become NP-complete. However, a few, in general PSPACE-complete, cases become polynomial time solvable for weakly acyclic input automata.

Related synchronization problems for weakly acyclic automata were previously investigated in [27. For example, in [27, it was shown that the problem to decide if a given subset of states could be mapped to a single state, a problem 
PSPACE-complete for general automata 2225], is NP-complete for weakly acyclic automata.

Furthermore, we investigate two problems related to subset synchronization, namely the problem if we can map the whole state set into a given target set by some word, and if we can map any given starting set into another target set. Both problems are PSPACE-complete in general [2|3|17|21|25|28]. However, for weakly acyclic automata the former becomes polynomial time solvable, as we will show here, and the latter becomes NP-complete.

Similar subset synchronization problems, for general, strongly connected and synchronizing automata, were investigated in [2].

Weakly acyclic automata are also known as partially ordered automata 6, or acyclic automata [19]. As shown in [6], the languages recognized by weakly acyclic automata are precisely the languages recognized by $\mathcal{R}$-trivial monoids.

\section{Preliminaries}

By $\Sigma$ we denote a finite set of symbols, also called an alphabet. By $\Sigma^{*}$ we denote the set of all words over $\Sigma$, i.e., finite sequences with the concatenation operation. The empty word is denoted by $\varepsilon$. A language $L$ is a subset $L \subseteq \Sigma^{*}$.

A partial deterministic finite automaton (PDFA) is denoted by a quintuple $\mathcal{A}=\left(\Sigma, Q, \delta, q_{0}, F\right)$, where $Q$ is a finite set of states, $\Sigma$ the input alphabet, $\delta: Q \times \Sigma \rightarrow Q$ is a partial transition function, $q_{0}$ the start state and $F \subseteq Q$ the set of final states. An automaton $\mathcal{A}=\left(\Sigma, Q, \delta, q_{0}, F\right)$ is called complete, if $\delta$ is a total function, i.e., $\delta(q, x)$ is defined for any $q \in Q$ and $x \in \Sigma$.

In the usual way, the transition function $\delta$ can be extended to a function $\hat{\delta}: Q \times \Sigma^{*} \rightarrow Q$ by setting, for $q \in Q, u \in \Sigma^{*}$ and $x \in \Sigma, \hat{\delta}(q, \varepsilon)=q$ and $\hat{\delta}(q, u x)=\delta(\hat{\delta}(q, u), x)$. In the following, we will drop the distinction between $\delta$ and $\hat{\delta}$ and will denote both functions simply by $\delta$.

For $S \subseteq Q$ and $u \in \Sigma^{*}$, we set $\delta(S, u)=\{\delta(s, u) \mid s \in S$ and $\delta(s, u)$ is defined $\}$ and $\delta^{-1}(S, u)=\{q \in Q \mid \delta(q, u)$ is defined and $\delta(q, u) \in S\}$. For $q \in Q$ and $u \in \Sigma^{*}$, we set $\delta^{-1}(q, u)=\delta^{-1}(\{q\}, u)$.

The language recognized by $\mathcal{A}$ is $L(\mathcal{A})=\left\{u \in \Sigma^{*} \mid \delta\left(q_{0}, u\right) \in F\right\}$.

We say that $q \in Q$ is reachable from $p \in Q$ (in $\mathcal{A}$ ) if there exists a word $u \in \Sigma^{*}$ such that $\delta(p, u)=q$.

For $\mathcal{A}=\left(\Sigma, Q, \delta, q_{0}, F\right)$ and $\Gamma \subseteq \Sigma$, by $\mathcal{A}_{\mid \Gamma}=\left(\Gamma, Q, \delta_{\mid \Gamma}, q_{0}, F\right)$ we denote the automaton $\mathcal{A}$ restricted to the subalphabet $\Gamma$, i.e., $\delta_{\mid \Gamma}: Q \times \Gamma \rightarrow Q$ with $\delta_{\mid \Gamma}(q, x)=\delta(q, x)$ for $q \in Q$ and $x \in \Gamma$.

We say a letter $x \in \Sigma$ induces a self-loop at a state $q \in Q$, if $\delta(q, x)=q$.

A state $s \in Q$ is called a sink state, if every letter induces a self-loop at it, i.e., $\delta(q, x)=q$ for any $x \in \Sigma$.

An automaton $\mathcal{A}=\left(\Sigma, Q, \delta, q_{0}, F\right)$ is called weakly acyclic, if it is complete and for any $q \in Q$ and $u \in \Sigma^{*} \backslash\{\varepsilon\}$, if $\delta(q, u)=q$, then $\delta(q, x)=q$ for any letter $x$ appearing in $u$, i.e., the simple ${ }^{2}$ cycles are self-loops. Equivalently, the reachability relation is a partial order. Here, we say a state $q$ is larger than another state

\footnotetext{
${ }^{2}$ A cycle is simple if it only involves distinct states [27.
} 
$p$, if $q$ is reachable from $p$ in $\mathcal{A}$. A state in a weakly acyclic automaton is called maximal, if it is maximal with respect to this partial order. Note that here, we require weakly acyclic automata to be complete. This is in concordance with $[27$. However, partially ordered automata are sometimes allowed to be partial in the literature [20. Equivalently, an automaton is weakly acyclic if and only if there exists an ordering $q_{1}, \ldots, q_{n}$ of its states such that if $\delta\left(q_{i}, x\right)=q_{j}$ for some letter $x \in \Sigma$, then $i \leqslant j$, i.e., we can topologically sort the states.

A semi-automaton $\mathcal{A}=(\Sigma, Q, \delta)$ is a finite complete automaton without a specified start state and with no specified set of final states. Every notion defined for complete automata that does not explicitly use the start state and the set of final states is also defined in the same way for semi-automata. For example, being weakly acyclic. When the context is clear, we call both finite automata and semi-automata simply automata.

A complete automaton $\mathcal{A}$ is called synchronizing if there exists a word $w \in \Sigma^{*}$ with $|\delta(Q, w)|=1$. In this case, we call $w$ a synchronizing word for $\mathcal{A}$. We call a state $q \in Q$ with $\delta(Q, w)=\{q\}$ for some synchronizing word $w \in \Sigma^{*}$ a synchronizing state.

For a fixed PDFA $\mathcal{B}=\left(\Sigma, P, \mu, p_{0}, F\right)$, we define the constrained synchronization problem:

Definition 1. $L(\mathcal{B})$-Constr-SYNC

Input: Deterministic semi-automaton $\mathcal{A}=(\Sigma, Q, \delta)$.

Question: Is there a synchronizing word $w$ for $\mathcal{A}$ with $w \in L(\mathcal{B})$ ?

The automaton $\mathcal{B}$ will be called the constraint automaton. If an automaton $\mathcal{A}$ is a yes-instance of $L(\mathcal{B})$-ConstR-SYNC we call $\mathcal{A}$ synchronizing with respect to $\mathcal{B}$. Occasionally, we do not specify $\mathcal{B}$ and rather talk about $L$-ConstR-SYNC. The unrestricted synchronization problem, i.e., $\Sigma^{*}$-ConstR-SYNC in our notation, is in $\mathrm{P}$ [30]. We are going to investigate this problem for weakly acyclic input automata only.

\section{Definition 2. $L(\mathcal{B})$-WAA-CONSTR-SYNC}

Input: Weakly acyclic semi-automaton $\mathcal{A}=(\Sigma, Q, \delta)$.

Question: Is there a synchronizing word $w$ for $\mathcal{A}$ with $w \in L(\mathcal{B})$ ?

We assume the reader to have some basic knowledge in computational complexity theory and formal language theory, as contained, e.g., in [18. For instance, we make use of regular expressions to describe languages. And we make use of complexity classes like P, NP, or PSPACE. The following was shown in [13.

Theorem 3 ([13]). Let $\mathcal{B}=\left(\Sigma, P, \mu, p_{0}, F\right)$ be a PDFA. If $|P| \leqslant 1$ or $|P|=2$ and $|\Sigma| \leqslant 2$, then $L(\mathcal{B})$-Constr-SynC $\in P$. For $|P|=2$ with $|\Sigma|=3$, up to symmetry by renaming of the letters, $L(\mathcal{B})$-CONSTR-SYNC is PSPACE-complete precisely in the following cases for $L(\mathcal{B})$ :

$$
\begin{array}{llll}
a(b+c)^{*} & (a+b+c)(a+b)^{*} & (a+b)(a+c)^{*} & (a+b)^{*} c \\
(a+b)^{*} c a^{*} & (a+b)^{*} c(a+b)^{*} & (a+b)^{*} c c^{*} & a^{*} b(a+c)^{*} \\
a^{*}(b+c)(a+b)^{*} & a^{*} b(b+c)^{*} & (a+b)^{*} c(b+c)^{*} & a^{*}(b+c)(b+c)^{*}
\end{array}
$$


and polynomial time solvable in all other cases.

In weakly acyclic automata, maximal states, sink states and synchronizing states are related as stated in the next lemmata.

Lemma 4. In a weakly acyclic automator ${ }^{3}$ a state is maximal if and only if it is a sink state.

Lemma 5. Let $\mathcal{A}=(\Sigma, Q, \delta)$ be a weakly acyclic automaton. If $\mathcal{A}$ is synchronizing, then the synchronizing state must be a unique sink state in $\mathcal{A}$ that is reachable from every other state and, conversely, such a state is a synchronizing state.

With Lemma 5, we can test if a given weakly acyclic automaton is synchronizing. First, check every state if it is a sink state. If we have found a unique sink state, then do a breadth-first search from this sink state by traversing the transitions in the reverse direction. This gives a better algorithm than the general algorithm, which runs in time $O\left(|\Sigma||Q|^{2}\right)$, see [30.

Corollary 6. For weakly acyclic automata we can decide in time $O(|\Sigma||Q|+|Q|)$ if it is synchronizing.

\section{Constrained Synchronization of Weakly Acyclic Automata}

In general, for any constraint automaton, the constrained synchronization problem is always in PSPACE, see 13. Here, we show that for weaky acyclic input automata, the constrained synchronization problem is always in NP. First, we establish a bound on the size of a shortest synchronizing word, which directly yields containment in NP as we have a polynomially bounded certificate which could be verified in polynomial time.

Proposition 7. Let $\mathcal{A}$ be a weakly acyclic automaton with $n$ states and $\mathcal{B}=$ $\left(\Sigma, P, \mu, p_{0}, F\right)$ be a fixed PDFA. Then, a shortest synchronizing word $w \in L(\mathcal{B})$ for $\mathcal{A}$ has length at most $|P|\left(\begin{array}{l}n \\ 2\end{array}\right)$.

Proof. Let $q_{1}, \ldots, q_{n}$ be a topological sorting of the states of $\mathcal{A}$. We represent the situation after reading a word $u \in \Sigma^{*}$, i.e., the set $\delta(Q, u)$, by a tuple $\left(i_{1}, \ldots, i_{n}\right) \in\{1, \ldots, n\}^{n}$, where $i_{j}$ is the index of $\delta\left(q_{j}, u\right)$ in the topological sorting, i.e., $\delta\left(q_{j}, u\right)=q_{i_{j}}$. Then, $u \in \Sigma^{*}$ is synchronizing if and only if the corresponding tuple is $(n, \ldots, n)$. The starting tuple is $(1, \ldots, n)$. For $\left(i_{1}, \ldots, i_{n}\right),\left(j_{1}, \ldots, j_{n}\right) \in\{1, \ldots, n\}^{n}$ we write $\left(i_{1}, \ldots, i_{n}\right)<\left(j_{1}, \ldots, j_{n}\right)$ if, for all $r \in\{1, \ldots, n\}$, we have $i_{r} \leqslant j_{r}$ and there exists at least one $s \in\{1, \ldots, n\}$ such that $i_{s}<j_{s}$.

${ }^{3}$ Recall that here, weakly acyclic automata are always complete. For partial automata such that the reachability relation is a partial order, this does not have to be true. 
Let $w=x_{1} \cdots x_{m} \in L(\mathcal{B})$ with $x_{i} \in \Sigma$ for $i \in\{1, \ldots, m\}$. Then, set $S_{i}=$ $\delta\left(Q, x_{1} \cdots x_{i}\right)$ and $S_{0}=Q$. Suppose $S_{i+|P|}=S_{i}$ for some $i \in\{0,1, \ldots, n\}$. Then, as $\mathcal{A}$ is weakly acyclid 4 , for the word $u=x_{i+1} \cdots x_{i+|P|}$ we have $\delta(q, u)=q$ for any $q \in S_{i}$ and, as it has length $|P|$, it induces a loop in the constraint automaton $\mathcal{B}$. So, we can replace this factor of $w$ by a shorter word $v \in \Sigma^{*}$ of length less than $|P|$ that yields the same result, i.e., $S_{i+|P|}=\delta\left(Q, x_{1} \cdots x_{i} v\right)$ and $x_{1} \cdots x_{i} v x_{i+|P|+1} \cdots x_{n} \in L(\mathcal{B})$.

Now, suppose $w=x_{1} \cdots x_{m} \in L(\mathcal{B})$ is a shortest synchronizing word for $\mathcal{A}$. By the previous paragraph, we can suppose $S_{i+|P|} \neq S_{i}$ for any $i \in\{1, \ldots, n\}$. As $\mathcal{A}$ is weakly acyclic, and we can only move forward in the topological sorting, if $\delta(Q, u) \neq \delta(Q, u v)$, then for the tuple $\left(i_{1}, \ldots, i_{n}\right)$ corresponding to $\delta(Q, u)$ and for the tuple $\left(j_{1}, \ldots, j_{n}\right)$ for $\delta(Q, u v)$ we have $\left(i_{1}, \ldots, i_{n}\right)<\left(j_{1}, \ldots, j_{n}\right)$. Note that we have equality if and only if $\delta(Q, u)=\delta(Q, u v)$. As we start with $(1, \ldots, n)$ and want to reach $(n, \ldots, n)$, we have to increase at least $n-1$ times the first entry, $n-2$ times the second and so on. Now, by the previous reasoning, every $|P|$ symbols we can suppose we increase some component. Combining these observations yields that a shortest synchronizing word has length at most

$$
|P| \cdot((n-1)+(n-2)+\ldots+1)=|P| \cdot\left(\begin{array}{l}
n \\
2
\end{array}\right) .
$$

This finishes the proof.

With Proposition 7 we can conclude that for weakly acyclic input automata, the constrained synchronization problem is always in NP.

Theorem 8, For weakly acyclic input automata and an arbitrary constraint automaton, the constrained synchronization problem is in NP.

\section{Subset Synchronization Problems}

Here, we will investigate the followig problems from [2]3/17/21/25|28/31] for weakly acyclic input automata.

Definition 9. SynC-From-SubSeT Input: $A=(\Sigma, Q, \delta)$ and $S \subseteq Q$. Question: Is there a word $w$ with $|\delta(S, w)|=1$ ?
Definition 10. SynC-InTO-SubSET Input: $A=(\Sigma, Q, \delta)$ and $S \subseteq Q$. Question: Is there a word $w$ with $\delta(Q, w) \subseteq S$ ?

\section{Definition 11. SetTransporter}

Input: $\mathcal{A}=(\Sigma, Q, \delta)$ and two subsets $S, T \subseteq Q$. Question: Is there a word $w \in \Sigma^{*}$ such that $\delta(S, w) \subseteq T$ ?

\footnotetext{
${ }^{4}$ More generally, it is also easy to see that in weakly acyclic automata, no word can induce a non-trivial permutation of a subset of states.
} 
These problems are PSPACE-complete in general 23 25128 for at least binary alphabets. In [27] it was shown that SYNC-FROM-SuBSET is NP-complete for weakly acyclic input automata. Interestingly, for weakly acyclic input automata, the complexity of SyNC-INTO-SUBSET drops considerably. Namely, we could solve the problem in polynomial time. Hence, the ability to have transitions that go backward seems to be essential to get hardness above polynomial time solvability for this problem.

Theorem 12, The problem SYNC-INTO-SUBSET is polynomial time solvable for weakly acyclic input automata. More generally 5 , given $S, T \subseteq Q$ such that $S$ contains all maximal states reachable from $S$, the existence of a word $w \in \Sigma^{*}$ such that $\delta(S, w) \subseteq T$ could be decided in polynomial time.

Not surprisingly, as SynC-From-SubSET is NP-complete 27. for at least binary alphabets, SETTRANSPORTER is NP-complete for at least binary alphabets.

Theorem 13. SetTransporter is NP-complete for weakly acyclic input automata when the alphabet is fixed but contains at least two distinct letters.

Proof. For containment in NP, suppose $(\mathcal{A}, S, T)$ with $\mathcal{A}=(\Sigma, Q, \delta), S, T \subseteq Q$, is an instance of SETTRANSPORTER with $\mathcal{A}$ being weakly acyclic. Let $a, b \notin \Sigma$ be two new symbols and $s_{f} \notin Q$ a new state. We can suppose $S, T$ are non-empty, for otherwise, if $S=\varnothing$ we have a trivial solution and if $S$ is non-empty and $T=\varnothing$ we have no solution at all. Then, construct $\mathcal{A}^{\prime}=\left(\Sigma \cup\{a, b\}, Q \cup\left\{s_{f}\right\}, \delta^{\prime}\right)$ with, for $q \in Q$ and $x \in \Sigma \cup\{a, b\}$,

$$
\delta^{\prime}(q, x)= \begin{cases}\delta(q, x) & \text { if } x \in \Sigma \\ s_{f} & \text { if } x=a \text { and } q \notin S \\ s_{f} & \text { if } x=b \text { and } q \in T \\ q & \text { otherwise. }\end{cases}
$$

and $\delta^{\prime}\left(s_{f}, x\right)=s_{f}$ for any $x \in \Sigma \cup\{a, b\}$. Note that $\delta^{\prime}\left(Q \cup\left\{s_{f}\right\}, a\right)=S \cup\left\{s_{f}\right\}$, $\delta^{\prime}(q, b)=s_{f}$ for $q \in Q$ if and only if $q \in T$ and that $\mathcal{A}^{\prime}$ is weakly acyclic as we have only added self-loops or transitions going into the sink state $s_{f}$. Then, there exists $w \in \Sigma^{*}$ such that $\delta(S, w) \subseteq T$ in $\mathcal{A}$ if and only if $\delta^{\prime}(Q, a w b)=\left\{s_{f}\right\}$ in $\mathcal{A}^{\prime}$. So, we have reduced the original problem to the problem to decide if $\mathcal{A}^{\prime}$ has a synchronizing word for the constraint language $a \Sigma^{*} b$. By Theorem 8 , the last problem is in NP.

For NP-hardness, we can use the same reduction as used in [27, Theorem 4] to show NP-hardness of SYNC-From-SuBSET with the same set $S$ but setting $T=\{f\}$, where $f$ is the sink state used in the reduction from [27].

In [17, it was shown that SetTransporter is NP-complete for general unary automata. For unary weakly acyclic automata, the problem is in P.

Proposition 14. If $|\Sigma|=1$, then SetTransporter is in $P$ for weakly acyclic input automata.

\footnotetext{
${ }^{5}$ This more general formulation was pointed out by an anonymous referee.
} 


\section{Constraint Automata with Two States and at most Three Letters}

Here, we give a complete classification of the complexity landscape of the constraint synchronization problem with weakly acyclic automata as input automata and when the constraint is given by an at most two state PDFA over an at most ternary alphabet.

For our NP-hardness result, we adapt a construction due to Eppstein and Rystsov [11|26] which uses the NP-complete SAT problem [8].

SAT

Input: $A$ set $X$ of $n$ boolean variables and a set $C$ of $m$ clauses;

Question: Does there exist an assignment of values to the variables in $X$ such that all clauses in $C$ are satisfied?

First, we single out those constraint languages that give NP-hard problems.

Proposition 15. For the following constraint languages, the constrained synchronization problem for weakly acyclic automata is NP-hard:

$$
\begin{array}{lll}
a(b+c)^{*} & (a+b+c)(a+b)^{*} & (a+b)(a+c)^{*} \\
(a+b)^{*} c(a+b)^{*} & a^{*} b(a+c)^{*} & a^{*}(b+c)(a+b)^{*} \\
a^{*} b(b+c)^{*} & (a+b)^{*} c(b+c)^{*} & a^{*}(b+c)(b+c)^{*}
\end{array}
$$

Proof (sketch). We only sketch the case $L(\mathcal{B})=(a+b)^{*} c(b+c)^{*}$, the other cases could be handled similarly. We adapt a reduction by Eppstein and Rystsov [11|26] to show NP-hardness for the decision variant of the problem of a shortest synchronizing word. Given a SAT instance with variables $X=\left\{x_{1}, \ldots, x_{n}\right\}$ and clauses $C=\left\{c_{1}, \ldots, c_{m}\right\}$, we construct a weakly acyclic automaton $\mathcal{A}=(\Sigma, Q, \delta)$ over the alphabet $\{a, b, c\}$ with states $q_{i, j}$ for $1 \leqslant i \leqslant m$ and $0 \leqslant j \leqslant n+1$, plus a sink state $q_{f}$. Then $\delta$ is defined, for $i \in\{1, \ldots, m\}$ and $j \in\{1, \ldots, n\}$, as

$$
\delta\left(q_{i, j}, b\right)= \begin{cases}q_{i, j+1} & \text { if } \bar{x}_{j} \in c_{i} \vee\left\{x_{j}, \bar{x}_{j}\right\} \cap c_{i}=\varnothing ; \\ q_{f} & \text { if } x_{j} \in c_{i} ;\end{cases}
$$

and, symmetrically,

$$
\delta\left(q_{i, j}, c\right)= \begin{cases}q_{i, j+1} & \text { if } x_{j} \in c_{i} \vee\left\{x_{j}, \bar{x}_{j}\right\} \cap c_{i}=\varnothing ; \\ q_{f} & \text { if } \bar{x}_{j} \in c_{i} .\end{cases}
$$

Furthermore, for $i \in\{1, \ldots, m\}$ and $j \in\{0, \ldots, n+1\}$,

$$
\delta\left(q_{i, j}, a\right)=\left\{\begin{array}{l}
q_{i, j} \text { if } j \in\{0,1\} ; \\
q_{f} \text { if } j \notin\{0,1\} .
\end{array}\right.
$$

Lastly, for $i \in\{1, \ldots, m\}$, we set $\delta\left(q_{i, n+1}, b\right)=\delta\left(q_{i, n+1}, c\right)=q_{i, n+1}, \delta\left(q_{i, 0}, b\right)=$ $q_{i, 0}, \delta\left(q_{i, 0}, c\right)=q_{i, 1}$ and $q_{f}=\delta\left(q_{f}, a\right)=\delta\left(q_{f}, b\right)=\delta\left(q_{f}, c\right)$. Note that we have $\left\{q_{1,1}, \ldots, q_{m, 1}\right\} \subseteq \delta(Q, u c)$ for any $u \in\{a, b\}^{*}$ and, for $v \in\{b, c\}^{*}, \delta\left(q_{i, 1}, v\right)=q_{f}$ 
if and only if some symbol in $v$ at a position smaller or equal than $n$ branches out of the strand $q_{i, 1}, \cdots, q_{i, n}$, which means $v$ could be identified with a satisfying assignment for the clause $c_{i}$. Conversely, if we have a satisfying assignment, construct a word $v=v_{1} \cdots v_{n} \in\{b, c\}^{*}$ by setting $v_{i}=b$ if the $i$-th variable is set to one, and $v_{i}=c$ otherwise. Then, $\delta(Q, a c v)=\left\{q_{f}\right\}$. So, we can show that $\mathcal{A}$ has a synchronizing word in $L(\mathcal{B})$ if and only if there exists a satisfying assignment for all clauses in $C$.

In the next two propositions, we handle those cases from the list given in Theorem 3 that do not appear in Proposition 15. It will turn out that for these cases, the complexity drops from PSPACE-completeness to polynomial time solvable.

Proposition 16. We have $\left((a+b)^{*} c\right)$-WAA-Constr-SynC $\in P$

Proof (sketch). By Lemma 5, if $\mathcal{A}$ is synchronizable, it must possess a unique synchronizing sink state $s_{f}$. In that case, set $T=\delta^{-1}\left(s_{f}, c\right)$. Then, we have a synchronizing word in $(a+b)^{*} c$ if and only if there exists a word $w \in(a+b)^{*}$ such that $\delta_{\mid\{a, b\}}(Q, w) \subseteq T$ in $\mathcal{A}_{\mid\{a, b\}}=\left(\Sigma, Q, \delta_{\mid\{a, b\}}\right)$. The latter problem is in $\mathrm{P}$ by Theorem 12 .

Proposition 17. We have $\left((a+b)^{*} c a^{*}\right)$-WAA-Constr-Sync $\in P$ and $((a+$ $b)^{*}\left(c^{*}\right)$-WAA-Constr-Sync $\in P$.

Proof (sketch). By Lemma 5, the automaton $\mathcal{A}$ could only be synchronizing if it has a unique sink state $s_{f}$. In this case, set $S_{i}=\delta^{-1}\left(s_{f}, a^{i}\right)$ and $n=|Q|$. We have $S_{i}=S_{n}$ for any $i \geqslant n$. Then, for each $i \in\{0, \ldots, n\}$, set $T_{i}=\delta^{-1}\left(S_{i}, c\right)$ and decide, which could be done in polynomial time by Theorem 12, if there exists a word $w \in\{a, b\}^{*}$ in $A_{\mid\{a, b\}}=\left(\{a, b\}, Q, \delta_{\mid\{a, b\}}\right)$ such that $\delta_{\mid\{a, b\}}(Q, w) \subseteq T_{i}$, which is equivalent to $\delta\left(Q, w c a^{i}\right)=\left\{s_{f}\right\}$.

Combining the results of this section, we can give a precise classification of the complexity landscape for the problem with weakly acyclic input automata and when the constraint automaton 6 has at most two states over a ternary alphabet.

Theorem 18, Let $\mathcal{B}=\left(\Sigma, P, \mu, p_{0}, F\right)$ be a PDFA. If $|P| \leqslant 1$ or $|P|=2$ and $|\Sigma| \leqslant 2$, then $L(\mathcal{B})$-WAA-Constr-SYNC $\in P$. For $|P|=2$ with $|\Sigma|=3$, up to symmetry by renaming of the letters, $L(\mathcal{B})$-WAA-CoNSTR-SYNC is NP-complete precisely for the cases listed in Proposition 15 and in $P$ otherwise.

\section{Relation to Automata with TTSPL Automaton Graphs}

In 445, the decision problem related to minimal synchronizing words was investigated for TTSPL automata. These are automata whose automaton graph, i.e., the multigraph resulting after forgetting about the labels, is a TTSPL graph,

${ }^{6}$ Recall that the constraint automaton is a partial automaton, whereas the input (semi-)automaton is always complete. 
i.e, a two-terminal series-parallel graph with a start and sink node and where self-loops are allowed.

In the context of automata theory, such automata were originally studied in connection with the size of resulting regular expressions, i.e., motivated by questions on the descriptional complexity of formal languages [14].

Many problems for series-parallel graphs are computationally easy [12, which partly motivated the aforementioned studies [45]. However, from a fixed parameter complexity perspective, for most parameters, synchronization problems remain hard on the corresponding automata class 445.

We will not give all the definitions, but refer the interested reader to the aforementioned papers. We only mention in passing that TTSPL automata form a proper subclass of the weakly acyclic automata. Also, by employing a similar construction as used in [4, Proposition 4.1], i.e., introducing two additional letters, an additional starting state and some auxiliary states to realize several paths from the start state by a tree-like structure to the starting states of the paths corresponding to the clauses in the reduction, we can alter the reduction from Proposition [15] to yield a TTSPL graph. However, we can even do better and note that for the reductions used in Proposition 15, we do not need additional letters, but can realize the branching from the additional starting state with two existing letters and use a third letter to map the additional states to the sink state. The resulting automaton is a TTSPL automaton, for example the transitions going directly to the sink state arise out of parallel compositions. Hence, we can even state the following.

Theorem 19. For the constrained synchronization problem restricted to input automata whose automaton graph is a TTSPL graph, we have the same classification result for small constraint PDFAs as stated in Theorem 18. In particular, we can realize NP-complete constrained problems.

\section{Conclusion}

We have investigated the complexity of the constrained synchronization problem for weakly acyclic input automata. We noticed that in this setting, the problem is always in NP. In the general setting, it was possible to have PSPACE-complete constrained problems, whereas this is no longer possibly in our setting. We have investigated the complexities for small constrained automata in the same way as done in the general case in 13. We found out that certain problems that are PSPACE-complete in general become NP-complete, whereas others that are PSPACE-complete even become polynomial time solvable. A similar phenomenon was observed for certain subset synchronization problems that are all PSPACEcomplete in general.

It is natural to continue this investigation for other classes of automata, to find out what properties are exactly needed to realize PSPACE-complete problems or for what other classes we only have NP-complete constrained problems, or what are the minimum requirements on the input automata to realize NPcomplete problems. 
Also, a complete classification of all possible realizable complexities, a problem orginally posed in [13, is still open. Hence, as a first step it would be interesting to know if for our restricted problem only the complexities $\mathrm{P}$ and NP-complete arise, or if we can realize a constrained problem equivalent to some NP-intermediate candidate problem.

Acknowledgement. I thank the anonymous reviewers for noticing some issues in the proofs of Theorem 13 and Proposition [15 that have been fixed. Also, I thank them for pointing out typos and some unclear formulations.

\section{References}

1. Amarilli, A., Paperman, C.: Topological sorting with regular constraints. In: Chatzigiannakis, I., Kaklamanis, C., Marx, D., Sannella, D. (eds.) ICALP 2018, July 9-13, 2018, Prague, Czech Republic. LIPIcs, vol. 107, pp. 115:1-115:14. Schloss Dagstuhl - Leibniz-Zentrum für Informatik (2018)

2. Berlinkov, M.V., Ferens, R., Szykula, M.: Preimage problems for deterministic finite automata. J. Comput. Syst. Sci. 115, 214-234 (2021)

3. Blondin, M., Krebs, A., McKenzie, P.: The complexity of intersecting finite automata having few final states. Comput. Complex. 25(4), 775-814 (2016)

4. Bruchertseifer, J., Fernau, H.: Synchronizing series-parallel automata with loops. In: Freund, R., Holzer, M., Sempere, J.M. (eds.) NCMA 2019, Valencia, Spain, July 2-3, 2019. pp. 63-78. Österreichische Computer Gesellschaft (2019)

5. Bruchertseifer, J., Fernau, H.: Synchronizing words and monoid factorization: A parameterized perspective. In: Chen, J., Feng, Q., Xu, J. (eds.) TAMC 2020, 16th International Conference, Changsha, China, October 18-20, 2020, Proceedings. Lecture Notes in Computer Science, vol. 12337, pp. 352-364. Springer (2020)

6. Brzozowski, J.A., Fich, F.E.: Languages of R-trivial monoids. J. Comput. Syst. Sci. 20(1), 32-49 (1980)

7. Černý, J.: Poznámka k homogénnym experimentom s konečnými automatmi. Matematicko-fyzikálny časopis 14(3), 208-216 (1964)

8. Cook, S.A.: The complexity of theorem proving procedures. In: Proceedings of the Third Annual ACM Symposium. pp. 151-158. ACM, New York (1971)

9. Diekert, V.: Makanin's algorithm for solving word equations with regular constraints. Report, Fakultät Informatik, Universität Stuttgart (03 1998)

10. Diekert, V., Gutiérrez, C., Hagenah, C.: The existential theory of equations with rational constraints in free groups is PSPACE-complete. Inf. Comput. 202(2), 105140 (2005)

11. Eppstein, D.: Reset sequences for monotonic automata. SIAM Journal on Computing 19(3), 500-510 (1990)

12. Eppstein, D.: Parallel recognition of series-parallel graphs. Inf. Comput. 98(1), 41-55 (1992)

13. Fernau, H., Gusev, V.V., Hoffmann, S., Holzer, M., Volkov, M.V., Wolf, P.: Computational complexity of synchronization under regular constraints. In: Rossmanith, P., Heggernes, P., Katoen, J. (eds.) MFCS 2019, August 26-30, 2019, Aachen, Germany. LIPIcs, vol. 138, pp. 63:1-63:14. Schloss Dagstuhl - Leibniz-Zentrum für Informatik (2019)

14. Gulan, S.: Series parallel digraphs with loops - graphs encoded by regular expression. Theory Comput. Syst. 53(2), 126-158 (2013) 
15. Gusev, V.V.: Synchronizing automata of bounded rank. In: Moreira, N., Reis, R. (eds.) Implementation and Application of Automata - 17th International Conference, CIAA. LNCS, vol. 7381, pp. 171-179. Springer (2012)

16. Hoffmann, S.: Computational complexity of synchronization under regular commutative constraints. In: Kim, D., Uma, R.N., Cai, Z., Lee, D.H. (eds.) Computing and Combinatorics - 26th International Conference, COCOON 2020, Atlanta, GA, USA, August 29-31, 2020, Proceedings. Lecture Notes in Computer Science, vol. 12273, pp. 460-471. Springer (2020)

17. Hoffmann, S.: On A class of constrained synchronization problems in NP. In: Cordasco, G., Gargano, L., Rescigno, A.A. (eds.) Proceedings of the 21st Italian Conference on Theoretical Computer Science, Ischia, Italy, September 14-16, 2020. CEUR Workshop Proceedings, vol. 2756, pp. 145-157. CEUR-WS.org (2020)

18. Hopcroft, J.E., Ullman, J.D.: Introduction to Automata Theory, Languages, and Computation. Addison-Wesley Publishing Company (1979)

19. Jirásková, G., Masopust, T.: On the state and computational complexity of the reverse of acyclic minimal dfas. In: Moreira, N., Reis, R. (eds.) Implementation and Application of Automata - 17th International Conference, CIAA 2012, Porto, Portugal, July 17-20, 2012. Proceedings. Lecture Notes in Computer Science, vol. 7381, pp. 229-239. Springer (2012)

20. Krötzsch, M., Masopust, T., Thomazo, M.: Complexity of universality and related problems for partially ordered nfas. Inf. Comput. 255, 177-192 (2017)

21. Luks, E.M., McKenzie, P.: Parallel algorithms for solvable permutation groups. J. Comput. Syst. Sci. 37(1), 39-62 (1988)

22. Martyugin, P.V.: Synchronization of automata with one undefined or ambiguous transition. In: Moreira, N., Reis, R. (eds.) Implementation and Application of Automata - 17th International Conference, CIAA. LNCS, vol. 7381, pp. 278-288. Springer (2012)

23. Pesant, G.: A regular language membership constraint for finite sequences of variables. In: Wallace, M. (ed.) CP 2004, Toronto, Canada, September 27 - October 1, 2004, Proceedings. LNCS, vol. 3258, pp. 482-495. Springer (2004)

24. Romeuf, J.: Shortest path under rational constraint. Inf. Process. Lett. 28(5), 245248 (1988)

25. Rystsov, I.K.: Polynomial complete problems in automata theory. Inf. Process. Lett. 16(3), 147-151 (1983)

26. Rystsov, I.K.: On minimizing the length of synchronizing words for finite automata. In: Theory of Designing of Computing Systems, pp. 75-82. Institute of Cybernetics of Ukrainian Acad. Sci. (1980), (in Russian)

27. Ryzhikov, A.: Synchronization problems in automata without non-trivial cycles. Theor. Comput. Sci. 787, 77-88 (2019)

28. Sandberg, S.: Homing and synchronizing sequences. In: Broy, M., Jonsson, B., Katoen, J.P., Leucker, M., Pretschner, A. (eds.) Model-Based Testing of Reactive Systems. LNCS, vol. 3472, pp. 5-33. Springer (2005)

29. Volkov, M.V.: Synchronizing finite automata. I. (in Russian, submitted)

30. Volkov, M.V.: Synchronizing automata and the Cerný conjecture. In: Martín-Vide, C., Otto, F., Fernau, H. (eds.) Language and Automata Theory and Applications, Second International Conference, LATA. LNCS, vol. 5196, pp. 11-27. Springer (2008)

31. Vorel, V.: Subset synchronization and careful synchronization of binary finite automata. Int. J. Found. Comput. Sci. 27(5), 557-578 (2016)

32. Vorel, V., Roman, A.: Complexity of road coloring with prescribed reset words. J. Comput. Syst. Sci. 104, 342-358 (2019) 


\section{A Proofs for Section 2 (Preliminaries)}

Lemma 4. In a weakly acyclic automaton ${ }^{7}$ a state is maximal if and only if it is a sink state.

Proof. As from a sink state no other state is reachable, it could not have any proper successor states, hence is maximal. Conversely, if a state is maximal, then by definition no other state is reachable from it, hence, every outgoing transition has to go back to this state, i.e., induces a self-loop.

Lemma 5. Let $\mathcal{A}=(\Sigma, Q, \delta)$ be a weakly acyclic automaton. If $\mathcal{A}$ is synchronizing, then the synchronizing state must be a unique sink state in $\mathcal{A}$ that is reachable from every other state and, conversely, such a state is a synchronizing state.

Proof. Let $\mathcal{A}=(\Sigma, Q, \delta)$ be weakly acyclic and $w \in \Sigma^{*}$ be such that $\delta(Q, w)=\{q\}$ for some $q \in Q$. Hence, $q$ is reachable from every other state and so must be maximal. By Lemma 4 $q$ is a sink state. Conversely, if we have a sink state $s \in Q$ reachable from every other state $q \in Q$ by a word $w_{q}$, we can construct a synchronizing word. We can suppose $Q$ has more than two states, for otherwise the problem is trivial. Set $w_{1}=w_{q}$ and $S_{1}=\delta\left(Q, w_{1}\right)$ for some $q \in Q \backslash\{s\}$. Then, inductively, let $i>1$ and, if $\left|S_{i-1}\right|>1$, choose $q \in S_{i-1} \backslash\{s\}$ and set $w_{i}=w_{i-1} w_{q}$. As $q$ and $s$ are mapped to $s \in S_{i-1}$, in this case $\left|S_{i}\right|<\left|S_{i-1}\right|$. So, after at most $|Q|-1$ many steps, for some $i \in\{1, \ldots,|Q|\}$ we must have $S_{i}=\{s\}$ and $\delta\left(Q, w_{i}\right)=\{s\}$.

Remark 1. Note that the procedure used in the proof of Lemma 5 works for any automaton with a sink state reachable from any other state.

\section{B Proofs for Section 3 (Constrained Synchronization of Weakly Acyclic Automata)}

Theorem [8, For weakly acyclic input automata and an arbitrary constraint automaton, the constrained synchronization problem is in NP.

Proof. By Proposition 7 we can guess a shortest synchronizing word in $L(\mathcal{B})$ of polynomial length. Verifying that such a word is indeed synchronizing could be done in polynomial time.

\section{Proofs for Section 4 (Subset Synchronization Problems)}

Theorem 12, The problem SynC-INTO-SUBSET is polynomial time solvable for weakly acyclic input automata. More generall $]^{8}$, given $S, T \subseteq Q$ such that $S$ contains all maximal states reachable from $S$, the existence of a word $w \in \Sigma^{*}$ such that $\delta(S, w) \subseteq T$ could be decided in polynomial time.

\footnotetext{
${ }^{7}$ Recall that here, weakly acyclic automata are always complete. For partial automata such that the reachability relation is a partial order, this does not have to be true.

8 This more general formulation was pointed out by an anonymous referee.
} 
Proof. We show the more general claim, the implication for SynC-InTO-SubSET is then implied by setting $S=Q$. Let $\mathcal{A}=(\Sigma, Q, \delta)$ be a weakly acyclic automaton. Let $R \subseteq S$ be the set of maximal states reachable from $S$ in $\mathcal{A}$ and suppose $R \subseteq S$ and $T \subseteq Q$. Set $n=|Q|$.

Claim: There exists $w \in \Sigma^{*}$ such that $\delta(S, w) \subseteq T$ if and only if $R \subseteq T$.

Proof of the Claim: By Lemma 4 the set $R$ only contains sink states. So, for any $w \in \Sigma^{*}$, we have $R \subseteq \delta(S, w)$. Hence, if $\delta(S, w) \subseteq T$, then $R \subseteq T$. Conversely, suppose $R \subseteq T$. Let $w_{\Sigma}$ contain every symbol from the input alphabet $\Sigma$ exactly once, in any order. Apart from the states in $R$, for every other state $q$ in $S$, there is a state $q^{\prime} \neq q$ that can be reached from $q$ by reading one symbol. As self-loops are the only cycles in weakly acyclic automata, we have $\delta\left(S, w_{\Sigma}^{n-1}\right) \subseteq R$. Hence, we find that $\delta\left(S, w_{\Sigma}^{n-1}\right) \subseteq T$. [End, Proof of the Claim]

Hence, we only have to check if $R \subseteq T$, which could be done in polynomial time, as $R$ is easily computable.

Proposition 14. If $|\Sigma|=1$, then SetTransporter is in $P$ for weakly acyclic input automata.

Proof. If $\mathcal{A}=(\{a\}, Q, \delta)$ is weakly acyclic over a unary alphabet, then it is a collection of paths that end in single state with a self-loops, where multiple paths could end in the same state labeled by a self-loop. Hence, for a given set $S \subset Q$, the states in $\delta\left(Q, a^{|Q|-1}\right)$ are precisely those states that are labeled by self-loops. Hence, $\delta\left(Q, a^{i}\right)=\delta\left(Q, a^{|Q|-1}\right)$ for all $i \geqslant|Q|$ and we only need to test the state sets $\delta\left(Q, a^{i}\right)$ for $i \in\{1, \ldots,|Q|-1\}$ if they equal a given target set $T \subseteq Q$. All these operations could be performed in polynomial time.

\section{Proofs for Section 5 (Constraint Automata with Two States and at most Three Letters)}

Proposition 15. For the following constraint languages, the constrained synchronization problem for weakly acyclic automata is NP-hard:

$$
\begin{array}{lll}
a(b+c)^{*} & (a+b+c)(a+b)^{*} & (a+b)(a+c)^{*} \\
(a+b)^{*} c(a+b)^{*} & a^{*} b(a+c)^{*} & a^{*}(b+c)(a+b)^{*} \\
a^{*} b(b+c)^{*} & (a+b)^{*} c(b+c)^{*} & a^{*}(b+c)(b+c)^{*}
\end{array}
$$

Proof. The following reductions are all adaptions of a reduction from Eppstein and Rystsov [Epp90,Rys80] to show NP-hardness for the decision variant of the problem of a shortest synchronizing word. The reduction works by constructing for every clause a linear path. The states on these paths correspond to the variables, and we can only leave this path and end in a synchronizing sink state at those states whose corresponding literals are contained in the clause associated to the path. The additional letter is used, in some sense, to reset all states either to the start states of the paths or to the sink state.

1. The constraint language $a(b+c)^{*}$. The adaption is similar to a reduction used in [Ryz19, Theorem 4]. 


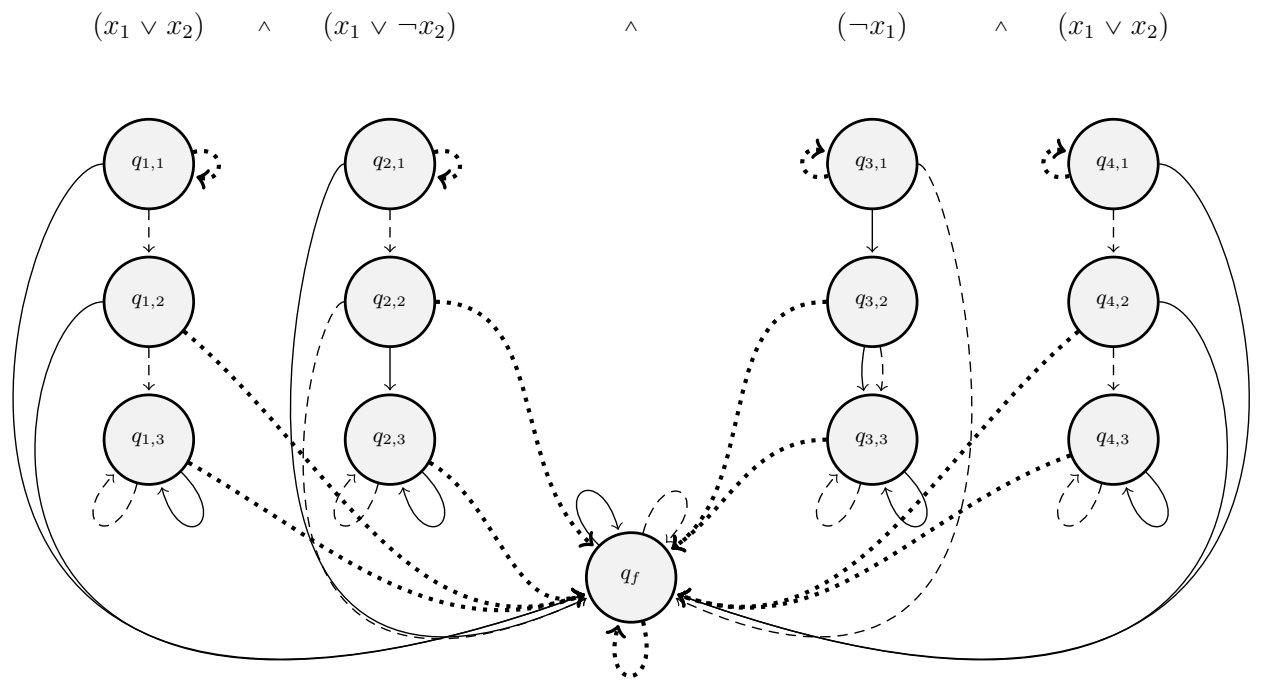

Fig. 1. Example for the SAT formula $\left(x_{1} \vee x_{2}\right) \wedge\left(x_{1} \vee \neg x_{2}\right) \wedge\left(\neg x_{1}\right) \wedge\left(x_{1} \vee x_{2}\right)$ of the reduction used in the proof of Proposition 15 for the constraint language $a(b+c)^{*}$. The transitions for $a$ are drawn with thick dotted lines, the transitions labeled by $b$ are drawn by dashed lines and those labeled by $c$ are drawn by solid lines.

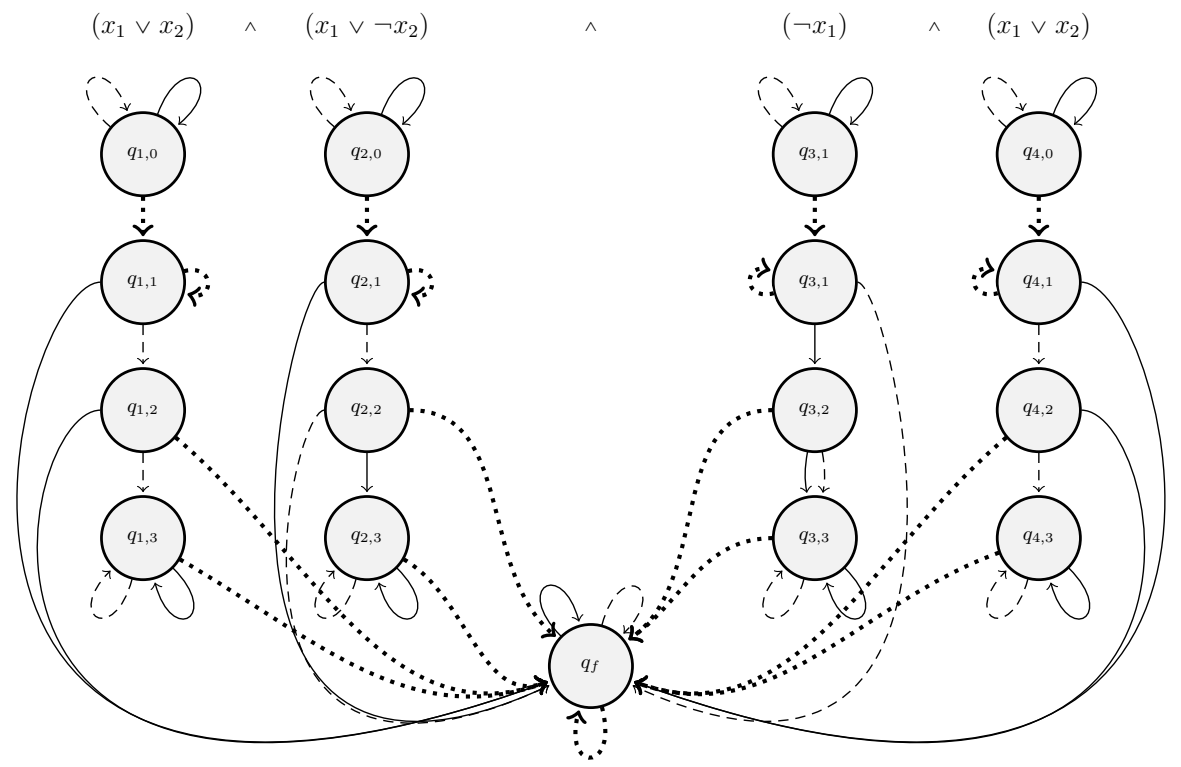

Fig. 2. A concrete example, for the SAT formula $\left(x_{1} \vee x_{2}\right) \wedge\left(x_{1} \vee \neg x_{2}\right) \wedge\left(\neg x_{1}\right) \wedge$ $\left(x_{1} \vee x_{2}\right)$, of the reduction used in the proof of Proposition 15 for $(a+b)^{*} c(a+b)^{*}$. The transitions for the letter $c$ are drawn with dotted lines, the transitions labeled by $a$ are drawn by dashed lines and those labeled by $b$ are drawn by solid lines. 


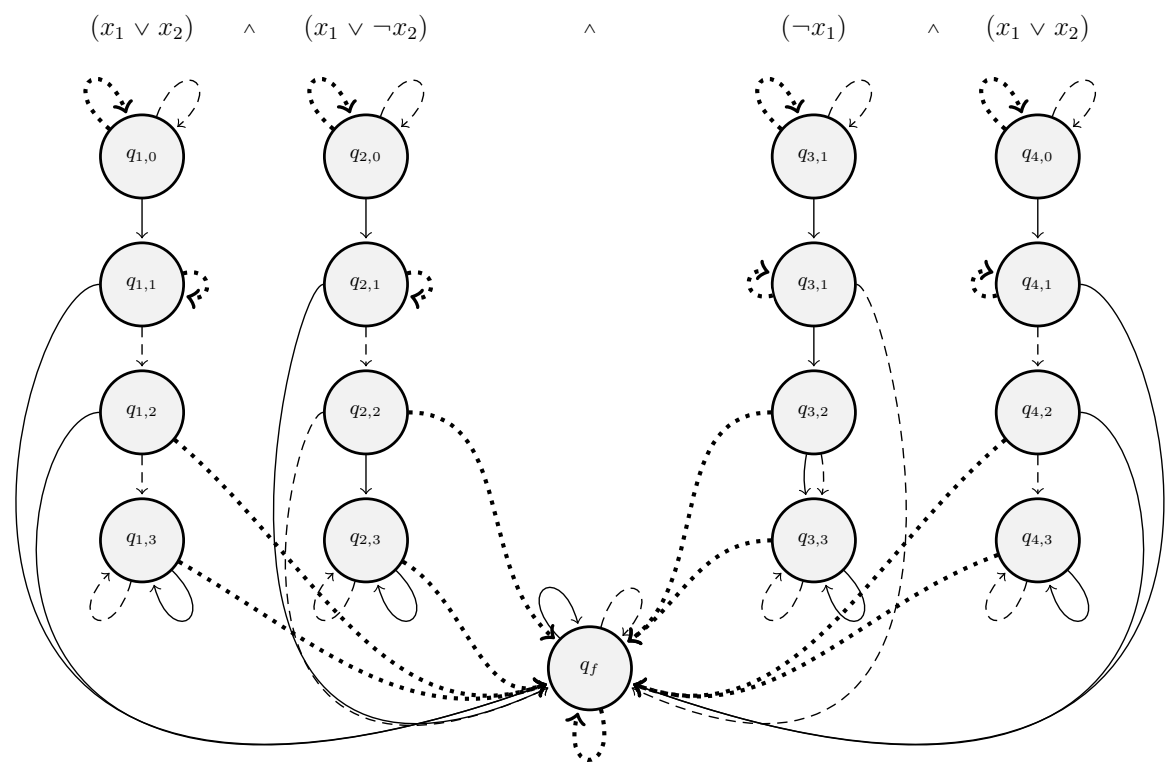

Fig. 3. Example for $\left(x_{1} \vee x_{2}\right) \wedge\left(x_{1} \vee \neg x_{2}\right) \wedge\left(\neg x_{1}\right) \wedge\left(x_{1} \vee x_{2}\right)$ of the reduction used in the proof of Proposition 15 for $(a+b)^{*} c(b+c)^{*}$. The $a$-transitions are drawn with dotted lines, the $b$-transitions by solid lines and the $c$-transitions by dashed lines. The self-loops at the $q_{i, 1}$ 's could also be changed to transitions ending in $q_{f}$.

Construction: Given a SAT instance with variables $X=\left\{x_{1}, \ldots, x_{n}\right\}$ and clauses $C=\left\{c_{1}, \ldots, c_{m}\right\}$, we construct a weakly acyclic automaton $\mathcal{A}=(\Sigma, Q, \delta)$ over the alphabet $\{a, b, c\}$ with states $q_{i, j}$ for $1 \leqslant i \leqslant m$ and $1 \leqslant j \leqslant n+1$, plus a sink state $q_{f}$. The transition function $\delta$ is defined, for $i \in\{1, \ldots, m\}$ and $j \in\{1, \ldots, n\}$, as

$$
\delta\left(q_{i, j}, b\right)= \begin{cases}q_{i, j+1} & \text { if } x_{j} \in c_{i} \vee\left\{x_{j}, \bar{x}_{j}\right\} \cap c_{i}=\varnothing ; \\ q_{f} & \text { if } \bar{x}_{j} \in c_{i} ;\end{cases}
$$

and, symmetrically,

$$
\delta\left(q_{i, j}, c\right)= \begin{cases}q_{i, j+1} & \text { if } \bar{x}_{j} \in c_{i} \vee\left\{x_{j}, \bar{x}_{j}\right\} \cap c_{i}=\varnothing ; \\ q_{f} & \text { if } x_{j} \in c_{i} .\end{cases}
$$

Furthermore, for $i \in\{1, \ldots, m\}$ and $j \in\{1, \ldots, n+1\}$, we set

$$
\delta\left(q_{i, j}, a\right)= \begin{cases}q_{i, 1} & \text { if } j=1 \\ q_{f} & \text { if } j \neq 0\end{cases}
$$

Lastly, for $i \in\{1, \ldots, m\}$, we set $\delta\left(q_{i, n+1}, b\right)=\delta\left(q_{i, n+1}, c\right)=q_{i, n+1}$, and $q_{f}=$ $\delta\left(q_{f}, a\right)=\delta\left(q_{f}, b\right)=\delta\left(q_{f}, c\right)$. See Figure 1 for an example of the reduction.

Verification: Suppose $\mathcal{A}$ has a synchronizing word $w \in L(B)$. Then, $w=a u$ with $u \in\{b, c\}^{*}$. As $q_{f}$ is a sink state, we have $\delta(Q, w)=\left\{q_{f}\right\}$. Consider a state $q_{i, 1}$. As $a$ labels a self-loop on this state, we have $\delta\left(q_{i, 1}, u\right)=s_{f}$. However, $\delta\left(q_{i, 1}, u\right)=s_{f}$ holds precisely if $u$ has a prefix $v \in\{b, c\}^{n}$ with $v=v_{1} \cdots v_{n}$ for $\left\{v_{1}, \ldots, v_{n}\right\} \subseteq \Sigma$ 
such that for any clause $c_{i}$ there exists $r \in\{1, \ldots, n\}$ with $v_{r}=b$ if $\bar{x}_{r} \in c_{i}$ or $v_{r}=c$ if $x_{r} \in c_{i}$. Hence, we get a satisfying assignment for all clauses by setting $x_{j}=0$ if $v_{j}=b$ and $x_{j}=1$ if $v_{j}=c$ for $j \in\{1, \ldots, n\}$.

Conversely, suppose a satisfying assignment for the variables $x_{1}, \ldots, x_{n}$ exists. Then, set $u=u_{1} \cdots u_{n}$ with $u_{j}=b$ if $x_{j}=0$ and $u_{j}=c$ if $x_{j}=1$ for $j \in\{1, \ldots, n\}$. Then, we have $\delta\left(q_{i, 1}, u\right)=s_{f}$ for $i \in\{1, \ldots, m\}$. As $\delta(Q, a)=\left\{q_{1,1}, \ldots, q_{m, 1}, q_{f}\right\}$, we find $\delta(Q, a u)=\left\{s_{f}\right\}$.

2. The constraint language $(a+b+c)(a+b)^{*}$. We can use the same reduction as in Case 1 but with the letters changed: the letter $c$ acts like the letter $a$ in Case 1 and the letter $a$ like the letter $c$ before. Note that we can use the letter $c$ if we have a satisfying assignment for the given SAT formula, but not conversely. However, by only investigating the paths taken from the start states $q_{i, 1}$, we can read of a satisfying assignment.

3. The constraint language $(a+b)(a+c)^{*}$. We can use the same reduction as in Case 1 but with the letters changed: the letter $b$ acts like the letter $a$ in Case 1 and the letter $a$ like the letter $b$ before. Note that we can use the letter $b$ if we have a satisfying assignment for the given SAT formula, but we do not need to use it in in the other direction of the reduction. However, by only investigating the paths taken from the start states $q_{i, 1}$, we can read of a satisfying assignment.

4. The constraint language $(a+b)^{*} c(a+b)^{*}$. Here, we need a different construction. The reason is that the special letter that is only allowed to be read once, which was the letter $a$ in Case 1 and is the letter $c$ here, had, in the previous cases, the property that before it, it was not possible to read anything, i.e., if it appeared in a word of the constraint language, it appeared as the first letter of that word. Without this property, for example in the case we are considering now, we can read in any word long enough to drive everything into the last states of the strands corresponding to the clauses, i.e., the states $q_{i, n+1}$ in the reduction of Case 1 Then, from this state we could read the special letter to map them to the the sink state.

We can circumvent this by introducing additional state $q_{i, 0}$. More formally, we give the complete construction next.

Construction: Given a SAT instance with variables $X=\left\{x_{1}, \ldots, x_{n}\right\}$ and clauses $C=\left\{c_{1}, \ldots, c_{m}\right\}$, we construct a weakly acyclic automaton $\mathcal{A}=(\Sigma, Q, \delta)$ over the alphabet $\{a, b, c\}$ with states $q_{i, j}$ for $1 \leqslant i \leqslant m$ and $0 \leqslant j \leqslant n+1$, plus a sink state $q_{f}$. The transition function $\delta$ is defined as, for $i \in\{1, \ldots, m\}$ and $j \in\{1, \ldots, n\}$,

$$
\delta\left(q_{i, j}, a\right)= \begin{cases}q_{i, j+1} & \text { if } x_{j} \in c_{i} \vee\left\{x_{j}, \bar{x}_{j}\right\} \cap c_{i}=\varnothing ; \\ q_{f} & \text { if } \bar{x}_{j} \in c_{i} ;\end{cases}
$$

and, symmetrically,

$$
\delta\left(q_{i, j}, b\right)= \begin{cases}q_{i, j+1} & \text { if } \bar{x}_{j} \in c_{i} \vee\left\{x_{j}, \bar{x}_{j}\right\} \cap c_{i}=\varnothing ; \\ q_{f} & \text { if } x_{j} \in c_{i} .\end{cases}
$$

Furthermore, for $i \in\{1, \ldots, m\}$ and $j \in\{1, \ldots, n+1\}$,

$$
\delta\left(q_{i, j}, c\right)= \begin{cases}q_{i, 1} & \text { if } j=1 \\ q_{f} & \text { if } j \neq 0\end{cases}
$$

Lastly, for $i \in\{1, \ldots, m\}$, we set $\delta\left(q_{i, n+1}, a\right)=\delta\left(q_{i, n+1}, b\right)=q_{i, n+1}$ and

$$
\delta\left(q_{i, 0}, a\right)=\delta\left(q_{i, 0}, b\right)=q_{i, 0}, \quad \delta\left(q_{i, 0}, c\right)=q_{i, 1}
$$


and $q_{f}=\delta\left(q_{f}, a\right)=\delta\left(q_{f}, b\right)=\delta\left(q_{f}, c\right)$.

Verification: Suppose $\mathcal{A}$ has a synchronizing word $w \in L(B)$. As $q_{f}$ is a sink state, we have $\delta(Q, w)=\left\{q_{f}\right\}$. Write $w=u c v$ with $u \in\{a, b\}^{*}$ and $v \in\{a, b\}^{*}$. For $i \in\{1, \ldots, m\}$, we have $\delta\left(q_{i, 0}, u c\right)=q_{i, 1}$. Hence, we must have $\delta\left(q_{i, 1}, v\right)=s_{f}$. By construction, this is the case precisely if $v$ has a prefix $v^{\prime} \in\{a, b\}^{n}$ with $v^{\prime}=v_{1}^{\prime} \cdots v_{n}^{\prime}$ for $\left\{v_{1}^{\prime}, \ldots, v_{n}^{\prime}\right\} \subseteq \Sigma$ such that for any clause $c_{i}$ there exists $r \in\{1, \ldots, n\}$ with $v_{r}^{\prime}=a$ if $\bar{x}_{r} \in c_{i}$ or $v_{r}^{\prime}=b$ if $x_{r} \in c_{i}$. Hence, we get a satisfying assignment for all clauses by setting $x_{j}=0$ if $v_{j}^{\prime}=a$ and $x_{j}=1$ if $v_{j}^{\prime}=b$ for all $j \in\{1, \ldots, n\}$.

Conversely, suppose a satisfying assignment for the variables $x_{1}, \ldots, x_{n}$ exists. Then, set $u=u_{1} \cdots u_{n}$ with $u_{j}=a$ if $x_{j}=0$ and $u_{j}=b$ if $x_{j}=1$ for $j \in$ $\{1, \ldots, n\}$. Then, $\delta\left(q_{i, 1}, u\right)=s_{f}$ for any $i \in\{1, \ldots, n\}$. Furthermore, we have $\delta(Q, c)=\left\{q_{1,1}, \ldots, q_{m, 1}, q_{f}\right\}$. Hence, $\delta(Q, c u)=\left\{s_{f}\right\}$.

5. The constraint language $a^{*} b(a+c)^{*}$. We can use the same reduction as in Case 4 but with the letter $b$ acting like the letter $c$ in Case 4 and the letter $c$ like the letter $b$ before.

6. The constraint language $a^{*}(b+c)(a+b)^{*}$. Here, we can use the same reduction as in Case 4 Note that the construction enforces that we have to use a word that uses the letter $c$.

7. The constraint language $a^{*} b(b+c)^{*}$. We can use the same reduction as in Case 4, but with the letter $b$ acting like the letter $c$ in Case 4 and the letter $c$ like the letter $b$ before.

8. The constraint language $(a+b)^{*} c(b+c)^{*}$. Here, the letter $a$ will be the letter that is used as a special letter. However, we can not use the previous constructions, but use another one. The construction was already given in the main text. Also, see Figure 3 for an example of the reduction.

Verification: Suppose $\mathcal{A}$ has a synchronizing word $w \in L(B)$. As $q_{f}$ is a sink state, we have $\delta(Q, w)=\left\{q_{f}\right\}$. Write $w=u c v$ with $u \in\{a, b\}^{*}$ and $v \in\{b, c\}^{*}$. For $i \in\{1, \ldots, m\}$, we have $\delta\left(q_{i, 0}, u c\right)=q_{i, 1}$. Hence, we must have $\delta\left(q_{i, 1}, v\right)=s_{f}$. By construction, this is the case precisely if $v$ has a prefix $v^{\prime} \in\{b, c\}^{n^{\prime}}, n^{\prime} \leqslant n$, with $v^{\prime}=v_{1}^{\prime} \cdots v_{n^{\prime}}^{\prime}$ for $\left\{v_{1}^{\prime}, \ldots, v_{n^{\prime}}^{\prime}\right\} \subseteq \Sigma$ such that for any clause $c_{i}$ there exists $r \in\{1, \ldots, n\}$ with $v_{r}^{\prime}=b$ if $x_{r} \in c_{i}$ or $v_{r}^{\prime}=c$ if $\bar{x}_{r} \in c_{i}$. Hence, we get a satisfying assignment for all clauses by setting $x_{j}=1$ if $v_{j}^{\prime}=b$ and $x_{j}=0$ if $v_{j}^{\prime}=c$ for all $j \in\left\{1, \ldots, n^{\prime}\right\}$, and setting the remaining variables to arbitrary values.

Conversely, suppose a satisfying assignment for the variables $x_{1}, \ldots, x_{n}$ exists. Then, set $u=u_{1} \cdots u_{n}$ with $u_{j}=b$ if $x_{j}=1$ and $u_{j}=c$ if $x_{j}=0$ for $j \in$ $\{1, \ldots, n\}$. Then, $\delta\left(q_{i, 1}, u\right)=s_{f}$ for any $i \in\{1, \ldots, n\}$. Furthermore, we have $\delta(Q, a c)=\left\{q_{1,1}, \ldots, q_{m, 1}, q_{f}\right\}$. Hence, $\delta(Q, a c u)=\left\{s_{f}\right\}$.

9. The constraint language $a^{*}(b+c)(b+c)^{*}$. We can use the same reduction as in Case 1

So, we have handled all constraint languages from the statement and the proof is done.

Proposition 16, We have $\left((a+b)^{*} c\right)$-WAA-Constr-Sync $\in P$

Proof. By Lemma 5] if $\mathcal{A}$ is synchronizable, it must possess a unique synchronizing sink state $s_{f}$, which is easily testable. So, if it does not possess a unique sink state, it could not have a synchronizing word in $(a+b)^{*} c$. If it has such a unique sink state $s_{f}$, 
set $T=\delta^{-1}\left(s_{f}, c\right)$. Then, we have a synchronizing word in $(a+b)^{*} c$ if and only if there exists a word $w \in(a+b)^{*}$ such that $\delta_{\mid\{a, b\}}(Q, w) \subseteq T$ in $\mathcal{A}_{\mid\{a, b\}}=\left(\Sigma, Q, \delta_{\mid\{a, b\}}\right)$. The latter problem is solvable in polynomial time by Theorem 12 and we have a polynomial time procedure for our original problem.

Proposition 17. We have $\left((a+b)^{*} c a^{*}\right)$-WAA-Constr-Sync $\in P$ and $\left((a+b)^{*} c c^{*}\right)$-WAA-Constr-SynC $\in$ $P$.

Proof. By Lemma 5 , the automaton $\mathcal{A}$ could only be synchronizing if it has a unique sink state $s_{f}$. Consider the sets $S_{i}=\delta^{-1}\left(s_{f}, a^{i}\right)$. As every path in $\mathcal{A}$ has length at most $|Q|$ and the only loops are self-loops, we have

$$
\left\{S_{i}: i \geqslant 0\right\}=\left\{S_{i}: i \in\{0, \ldots,|Q|\}\right\} .
$$

More specifically, as paths of length $|Q|$ or more induce a (self-)loop, we have $\delta\left(q, a^{|Q|+1}\right)=$ $s_{f}$ if and only if $\delta\left(q, a^{|Q|}\right)=s_{f}$, which yields $S_{|Q|+1}=S_{|Q|}$ and so, inductively, $S_{i}=S_{|Q|}$ for all $i \geqslant|Q|$. Then, for each $i \in\{0, \ldots,|Q|\}$, set $T_{i}=\delta^{-1}\left(S_{i}, c\right)$ and decide if there exists a word $w \in\{a, b\}^{*}$ in $A_{\mid\{a, b\}}=\left(\{a, b\}, Q, \delta_{\mid\{a, b\}}\right)$ such that $\delta_{\mid\{a, b\}}(Q, w) \subseteq T_{i}$. By Theorem 12 the last step could be done in polynomial time and as we only have to perform this step $|Q|+1$ many times and the sets $T_{i}$ and $S_{i}$ are computable in polynomial time, the whole procedure runs in polynomial time.

Theorem 18. Let $\mathcal{B}=\left(\Sigma, P, \mu, p_{0}, F\right)$ be a PDFA. If $|P| \leqslant 1$ or $|P|=2$ and $|\Sigma| \leqslant 2$, then $L(\mathcal{B})$-WAA-Constr-SYNC $\in P$. For $|P|=2$ with $|\Sigma|=3$, up to symmetry by renaming of the letters, $L(\mathcal{B})$-WAA-CONSTR-SYNC is NP-complete precisely for the cases listed in Proposition [15] and in $P$ otherwise.

Proof. By Theorem 3 , for at most two states and a binary alphabet, the problem is always in P. Up to symmetry, for $|P|=2$ and $|\Sigma|=3$ we only have to check the cases listed in Theorem 3 as for the other ones it is in $\mathrm{P}$ for general input automata. Except for the cases the cases

$$
(a+b)^{*} c, \quad(a+b)^{*} c a^{*} \text { and }(a+b)^{*} c c^{*},
$$

these are all listed in Proposition 15 And for the cases from Theorem 3 not appearing in Proposition [15] written above we have polynomial time solvable problems by Proposition 16 and Proposition 17. Containment in NP is stated in Theorem 8

\section{References for the Appendix}

Epp90. D. Eppstein. Reset sequences for monotonic automata. SIAM Journal on Computing, 19(3):500-510, 1990.

Rys80. Igor K. Rystsov. On minimizing the length of synchronizing words for finite automata. In Theory of Designing of Computing Systems, pages 75-82. Institute of Cybernetics of Ukrainian Acad. Sci., 1980. (in Russian).

Ryz19. Andrew Ryzhikov. Synchronization problems in automata without non-trivial cycles. Theor. Comput. Sci., 787:77-88, 2019. 